\title{
Labyrinthe
}

\section{Le dispositif sériel}

Sur un trailer des Sopranos, saison $6,2^{\mathrm{e}}$ partie.

\section{Renaud Pasquier}

\section{(2) OpenEdition}

Journals

Édition électronique

URL : http://journals.openedition.org/labyrinthe/4188

DOI : $10.4000 /$ labyrinthe.4188

ISSN : 1950-6031

Éditeur

Hermann

\section{Édition imprimée}

Date de publication : 15 août 2011

Pagination : 41-45

ISBN : 9782705681470

Référence électronique

Renaud Pasquier, «Le dispositif sériel », Labyrinthe [En ligne], 37 | 2011 (2), mis en ligne le 01 août 2013, consulté le 20 avril 2019. URL : http://journals.openedition.org/labyrinthe/4188 ; DOI : 10.4000/ labyrinthe. 4188 


\section{Le dispositif sériel}

\section{Sur un trailer des Sopranos, saison 6, $2^{\mathrm{e}}$ partie.}

Renaud Pasquier

La vidéo commentée dans ce texte est consultable à cette adresse: http://www.youtube.com/watch?v=B81HGw4arsM

Cette vidéo présentant les derniers épisodes de la série (trailer est le terme consacré), ceux de la sixième et dernière saison, est un objet singulier: nulle voix off qui annonce la teneur des nouveaux épisodes, pas plus de rappels explicites des saisons précédentes, pas de scènes passées ou à venir, pas de lieu emblématique, enfin, ni véritablement identifiable. Certes, le trailer en question est conçu avant tout pour les spectateurs qui connaissent déjà la série, et se passent aisément de résumés ou d'allusion. Ces cinquante-neuf secondes n'en demeurent pas moins extrêmement dépouillées, voire épurées, comme pensées pour mettre à distance toute familiarité avec la fiction re-présentée. L'inverse du trailer traditionnel, en somme.

Que voit-on? Un paysage indécis, intermédiaire, entre terre et eau, désert, ou presque, peu de végétation, des roseaux, épars, comme les nuages dans le ciel crépusculaire (matin? soir? on ne sait). Sur la rive, aussi près de l'eau que possible - on distinguera progressivement sous ses pieds une avancée, une jetée mangée par l'eau et la mousse, qui évoque irrésistiblement une planche de condamné, sur un bateau pirate - un corps massif, vu de dos, vêtu d'un long manteau noir, large et ténébreuse silhouette qui s'épaissit à mesure que la caméra s'en approche, imposant sa lourde présence à la terre, à l'eau, au ciel; on devine Tony Soprano dans cette figure opaque - opaque, qui le reconnait sait que le personnage l'est plus que jamais après six saisons, les ressorts réels de ses tourments et de ses motivations restant désespérément flous, malgré sa quasi omniprésence à l'écran, son occupation opiniâtre du plan. 
Son immobilité ne laisse pas d'intriguer - l'écart entre les jambes assure un équilibre impeccable, inébranlable, minéral - il ne se passe rien, rien qu'un vent timide qui agite très légèrement les pans du manteau, et ride à peine la surface des eaux (comme bien souvent rien n'advient dans Les Sopranos, entre un bain de sang et une querelle familiale). Que vient-il faire ici? Simplement se recueillir, loin du bruit et de la fureur qu'il régente quotidiennement, contempler et écouter les canards sauvages? S'agit-il d'un rendez-vous d'affaires dans un coin tranquille, judicieusement choisi? Est-ce la scène d'un crime à venir? D'un suicide, pourrait-on se demander devant le visage fermé, contracté, de Tony, quand un prudent mouvement de caméra nous le découvre? Est-ce bien de prudence qu'il faut parler, la caméra craint-elle d'approcher Tony? Conserve-t-elle une distance respectueuse? À moins qu'au contraire elle ne le menace sournoisement, tentée de le jeter à l'eau? La paranoïa est permise, elle est même sollicitée dans ce décor où l'œil, agacé de glisser sur une image sans aspérités, est tenté d'aller les chercher, d'attribuer une intensité inouïe au détail le moins saillant - ou comment l'insignifiant se fond dans le tout-signifiant.

Est-ce justement pour cela, happé par l'indifférence souveraine de cette courte séquence, que je tends à y voir une sorte de critique interne de la série, une mise à nu de son dispositif, voire du dispositif de toute série? Quand bien même telle interprétation serait secrétée par mon cerveau aux aguets, séquelle d'une irritation née du frottement avec les images, un délire, en somme, cela, pour la fragiliser, ne l'invalide en rien, dès lors qu'elle est bien le produit d'une expérience de spectateur, qu'elle est le fruit d'un long commerce avec la fiction concernée. Persévérons alors.

Les Sopranos, c'est donc un corps, celui de Tony. C'est lui qui assure l'unité et la continuité des six saisons, le singulier qui sous-tend le pluriel du titre. Mais toute série ne se définit et ne peut perdurer qu'en articulant le Même et l'Autre, ou plus exactement l'Un et le Multiple: nécessité narrative, thématique, commerciale autant qu'artistique. Le Multiple est bien présent dans le trailer: il s'incarne dans les voix qui le peuplent et le scandent, prenant vite le pas sur les caquètements initiaux, voix de femmes et d'hommes, répliques de personnages empruntées au six saisons qui se sont écoulées. 
Des voix, des phrases, des volutes de mots, qu'on voit presque s'élever lentement, se nouer les unes aux autres en un réseau qui peu à peu enveloppe le large corps immobile, et ce avant même de démêler le sens des mots et les identités des locuteurs, longue phrase chorale, alternant segments longs et brefs, au rythme d'abord lent, solennel, tant que les timbres sont uniquement féminins, puis de plus en plus rapide, nerveux, et à mesure que les voix se virilisent, le ton s'hystérise, s'exaspère, avant l'accalmie finale qui n'est en rien un apaisement, la séquence se clôt par la voix même qui l'ouvrait, mais là où elle était tremblante, percluse d'émotions, elle est à présent sèche et métallique, définitive: Everything comes to an end. Tony sort du champ.

Cette voix encadrante, c'est celle de Carmela, son épouse, celle qui justifie au mieux le pluriel du titre, la seule qui parvient à contester l'omniprésence de Tony à l'écran. Car telle est bien la dynamique de la fiction, la tension entre l'Un et le Multiple qui anime la série: prendre la place de Tony, ou tout au moins se faire une place auprès de lui, tandis que tous ses actes tendent à maintenir et prolonger indéfiniment le règne de l'Un, le sien. Le Multiple est à la fois une nécessité (sur qui régner, qui dominer, sinon?) et une menace, à réprimer donc sans pitié ni hésitation. C'est aussi une souffrance, dès lors qu'il faut l'affronter non seulement à l'extérieur, mais aussi en soi-même. Découvrir en soi non seulement l'Autre, mais, pire encore, les Autres, alors même qu'on s'efforce de maintenir l'Unité et l'Unicité de son propre pouvoir, voilà une source d'angoisse infinie, qui conduit logiquement Tony en thérapie.

Les voix parlent à Tony, elles l'habitent, le hantent, le harcèlent. Toutes ces phrases, que le spectateur assidu (et anglophone) attribuera sans coup férir à tel personnage dans telle situation, elles pourraient au fond être prononcées par Tony, composantes de son polyphonique for intérieur (où l'on n'oublie pas que for signifie en latin « je parle »). "Je veux t'ouvrir mon cœur", et Tony qui se révèle à nous, du moins partiellement, pendant six saisons, et durant ces cinquante-neuf secondes; "La dépression, c'est de la rage tournée vers l'intérieur », pour preuve la colère qui déborde seconde après seconde de ce discours interne; «Je suis le Boss, Bon Dieu! », « Je veux ce à quoi j'ai droit! » « Je donne ma vie, pour ce truc! », trois voix différentes, trois affirmations assignables 
à Tony, son Ego hypertrophié, son appétit de pouvoir et de possession, sa fatigue devant l'exigence toujours croissante de ces pulsions...

Les voix, les phrases, les prières, menaces, interrogations, insultes, confessions, etc., sont pourtant celles de Carmela, sa femme, de Livia Soprano, sa mère, de Jennifer Melfi, sa psychothérapeute, de Meadow, sa fille, de Chris, son neveu, de Junior, son oncle, de Pussy, son ancien meilleur ami, de Ralph Cifaretto, de Johnny Sack, de Richie Aprile, tous affranchis, tous tour à tour alliés et adversaires de Tony... Et s'il les emprunte dans son introspection, c'est qu'il se les est appropriées mais c'est aussi qu'elles-mêmes ont pris racine en lui, se sont installées dans son être, dont elles sont à présent partie intégrante.

Ce qui vaut pour Tony Soprano vaut encore plus pour James Gandolfini, son interprète. La fatigue qui marque son visage est aussi celle de l'acteur, las d'incarner depuis des années le mafieux névrosé, ses propres souvenirs rongés, parasités par ceux de Tony, les noms des acteurs et ceux des personnages s'entremêlant, noués par ces visages et ces voix identiques, et sans doute balance-t-il entre soulagement et tristesse à l'idée d'enfin se défaire de Tony Soprano, ou plutôt d'en cesser l'incarnation, de le convertir en un inoffensif spectre qui sans doute subsistera plus ou moins longtemps, flottant légèrement dans telle strate de son esprit, mais sera bien vite chassé, relégué dans un lointain repli par de nouvelles incarnations, de nouveaux spectres. Mais si cela est vrai pour l'acteur, cela l'est aussi pour le spectateur: c'est bien sûr à lui, c'est-à-dire à nous, que s'adresse le catégorique «Everything comes to an end » énoncé par Carmela, nous qui ressentons la douleur et le soulagement de cette fin crainte et désirée, nous qui nous répétons en sanglotant devant notre écran, le décompte fait des heures passées à le fixer, "I'm giving my life for this thing ", nous qui entendons monter en nous les bribes de phrases et d'images prélevées par notre mémoire sur les soixante-dix-sept épisodes (et à peine moins d'heures) déjà vus.

Ce n'est donc pas seulement le dispositif portant des Sopranos que met si exactement en scène ce trailer, c'est aussi l'une des puissances d'attraction spécifique de la série comme forme esthétique: non pas la gestion savante des péripéties et le suspense qui en procède, non plus l'identification, si souvent invoquée comme l'alpha et l'oméga du plaisir fictionnel, mais cette communauté affectuelle élaborée entre acteurs, 
personnages, et spectateurs, qui ne relève en rien de l'identité (fût-elle fantasmatique) mais bien plutôt de l'analogie et du partage d'une expérience, celle de la pluralité vécue. Je ne m'identifie pas à Tony, mais je partage avec lui cette auto-construction à partir de bribes pillées çà et là et recomposées perpétuellement, en une élaboration sans fin (ou pas tout à fait, car hélas, everything comes to an end...) où la frontière entre fiction et réalité n'existe pas, tout est matière, tout est bon. Si Tony est privilégié, ce n'est pas, là non plus, en tant qu'individu identifié, mais en tant qu'il occupe une position privilégiée, centrale (et entend bien la maintenir) dans un dispositif précisément centripète, au carrefour de toutes les trajectoires, tous les affects, tous les regards.

Si donc le motif de la double vie, du secret, des tourments intimes qui déchirent l'âme (Tony, mais aussi Don Draper, le génial publicitaire de Mad Men qui cache son véritable nom, Nate Fisher, dans Six Feet Under, qui au cours d'une opération qui le plonge dans le coma, et d'une séquence magnifique, visite les pièces de la demeure familiale comme autant de vies possibles, de versions de lui-même, ou encore Dexter, le serial killer tueur de serial killers), si les personnages protéiformes, s'inventant histoires et statuts différents à chaque épisode (Code Quantum, Le Caméléon, Dollhouse, trois séries qui reposent sur un principe scénaristique commun, celui d'un héros aux facultés extraordinaires plongé à chaque épisode dans une situation, des problèmes, et une vie différentes), voire les figures de possédés (Twin Peaks) ou de schizophrènes (United States of Tara) hantent les séries télévisées, ce n'est pas seulement parce qu'ils offrent des ressources scénaristiques particulièrement séduisantes ou parce qu'ils permettent des performances d'acteur. C'est avant tout parce qu'ils actualisent la tension intime propre au dispositif sériel, parce qu'ils mettent en lumière son mode d'action le plus orignal: la série est un puissant appareil de désidentification. À travers elle, je ne suis plus moi, je suis les autres; je suis nous, je suis vous, je suis tous. 
